\title{
Integrated Quantum Dot Barcode Smartphone Optical Device for Wireless Multiplexed Diagnosis of Infected Patients
}

\author{
Kevin Ming, Jisung Kim, Mia J. Biondi, Abdullah Syed, Kun Chen, \\ Albert Lam, Mario Ostrowski, Anu Rebbapragada, Jordan J. Feld, \\ and Warren C. W. Chan
}

Version Post-Print/Accepted Manuscript

Citation K. Ming, J. Kim, M. J. Biondi, A. Syed, K. Chen, A. Lam, M. Ostrowski, (published version) A. Rebbapragada, J. J. Feld, W. C. W. Chan, "Integrated Quantum Dot Barcode Smartphone Optical Device for Wireless Multiplexed Diagnosis of Infected Patients," ACS Nano, 2015 (Mar), 9, 3060. $\mathrm{JIF}=12.0$.

Publisher's Statement This document is the Accepted Manuscript version of a Published Work that appeared in final form in ACS Nano, copyright (CAmerican Chemical Society after peer review and technical editing by the publisher. To access the final edited and published work see http://dx.doi.org/10.1021/nn5072792. 


\section{An Integrated Quantum Dot Barcode Smartphone}

\section{Optical Device for Wireless Multiplexed}

\section{Diagnostics}

Kevin Ming ${ }^{1,2, \$}, J_{i s u n g}$ Kim $^{1,2, \$}$, Mia J. Biondi ${ }^{8}$, Abdullah Syed ${ }^{1,2}$, Kun Chen $^{1,2}$, Albert Lam ${ }^{1,2}$, Mario Ostrowski ${ }^{6}$, Anu Rebbapragada ${ }^{7}$ Jordan J. Feld ${ }^{8}$, Warren C. W. Chan ${ }^{1,2,3,4,5}$

${ }^{1}$ Institute of Biomaterials and Biomedical Engineering, ${ }^{2}$ Terrence Donnelly Centre for Cellular and Biomolecular Research, ${ }^{3}$ Chemistry, ${ }^{4}$ Chemical Engineering, ${ }^{5}$ Materials Science and Engineering, ${ }^{6}$ Department of Immunology, ${ }^{7}$ Laboratory Medicine and Pathobiology,${ }^{8}$ Sandra Rotman Centre for Global Health, University of Toronto, 160 College Street, Toronto, ON, M5S 3E1, Canada. $¥$ These authors contributed equally.

Corresponding Author: Dr. Warren C.W. Chan.

Warren.chan@utoronto.ca

416-946-8416

Institute of Biomaterials and Biomedical Engineering,

160 College Street, Rm 402 Toronto, ON, M5S 3E1, Canada.

Ming et al. Chan 


\section{KEYWORDS}

quantum dot barcode, smartphone, point-of-care, infectious disease, diagnostics

\section{ABSTRACT}

Inorganic nanoparticles are ideal precursors for engineering barcodes for rapidly detecting diseases. Despite advances in the chemical design of these barcodes, they have not advanced to clinical use because they lack sensitivity and are not cost-effective due to requirement of a large read-out system. Here we combined recent advances in quantum dot barcode technology with

10 smartphones to engineer a simple and low-cost chip-based wireless multiplex diagnostic device. We characterized the analytical performance of this device and demonstrated the device is capable of detecting down to 1000 viral genetic copies per $\mathrm{mL}$ and this enabled the diagnosis of patients infected with HIV or hepatitis B. More importantly, the barcoding enabled us to detect multiple infectious pathogens (e.g., HIV and hepatitis B) simultaneously, in a single test, in less

15 than 1 hour. This multiplexing capability of the device enables the diagnosis of infections that are difficult to differentiate clinically due to common symptoms such as a fever or rash. The integration of quantum dot barcoding technology with a smartphone reader provides a capacity for global surveillance of infectious diseases and the potential to accelerate knowledge exchangetransfer of emerging or exigent disease threats with healthcare and military organizations in real20 time.

Ming et al. Chan 


\section{TEXT}

The detection of diseases requires the development of technologies that can rapidly analyze

25 genetic and proteomic targets with a single drop of biological fluid. The detection assays must be cost-effective and detect multiple targets simultaneously which would increase accessibility and improve the speed of analysis, respectively. Ultimately, this leads to improvements in the diagnostic sensitivity and specificity and would contain the spread of diseases, especially for infectious pathogens. In the last decade, researchers have developed a wide-array of barcoding

30 structures and have demonstrated the detection of multiple biological targets in buffer. The barcodes comprise of graphical, ${ }^{1,2}$ optical,,${ }^{3,4}$ or magnetic ${ }^{5,6}$ structures with unique patterns that identify surface coated bio-recognition molecules which can selectively recognize biological targets of interest (e.g. whole virus, antigen, or genetic sequence). Detection occurs when a fluorescently labelled secondary targeting agent is added to the barcode, forming a sandwich

35 structure. The code identifies the coated recognition molecule, which identifies the target; and the secondary probe confirms the presence of the disease and the quantity. The advantage of using barcode in a diagnostic setting is that multiple targets from patient samples can be detected simultaneously which increase the speed of analysis and improve the precision and accuracy of diagnosis. Despite advances with the chemical design of barcodes,

40 nanotechnology-based barcoding technologies have not advanced to patient care. The read-out device (e.g. spectrophotometer/fluorometer, fluorescent microscope, or flow cytometer) remains expensive, and the poor sensitivity (limit of detection of femto to attomole) limits their utility for detecting most clinically relevant samples. While this can be overcome with amplification steps, current approaches are often too complex or expensive to be practical outside of research laboratories. Here, we have combined recent advances in quantum dot barcoding technology, Ming et al. Chan 
smartphones, amplification, and software design to engineer a diagnostic device capable of detecting multiple pathogens (Figure 1) in patient samples. This work is, to the best of our knowledge, the first to demonstrate the use of a portable, smartphone-based read-out of quantum dot barcodes, which enhances their ability and practically for use in point-of-care diagnostics.

Barcodes can be engineered by infusing polystyrene beads with different emitting quantum dots. ${ }^{7,8}$ The quantum dots provide significant advantages over organic fluorophores for barcoding. They are brighter, have wider excitation spectra, narrower emission spectra, and are more resistant to photobleaching. ${ }^{9-11}$ From a device perspective, these properties are attractive because they reduce the costs of manufacturing the diagnostic device and simplify the design of the read-out system while giving high precision in identifying the optical code. This strategy also provides quantum dots with greater multiplexing capabilities, as the use of quantum dot in a non-barcoded detection system can reasonably differentiate 5-6 unique signals ${ }^{10,11}$ versus the potentially millions in a barcode format. ${ }^{7}$ Furthermore, the optical signals of the quantum dots are protected in the microbead from fluorescence fluctuations as the polystyrene reduces the interaction of the quantum dots with ions, proteins, and other biological molecules. ${ }^{12,13}$ The microbeads themselves are also versatile in molecular detection and can detect both genomic or proteomic targets. ${ }^{7,14,15}$ We have previously optimized the methods to prepare quantum dot barcodes with hundreds of distinct optical patterns and demonstrated their utility as a universal

65 platform for detecting different panels of infectious targets using model synthetic genomic sequences. ${ }^{16}$ A critical next step is then to improve the analytical sensitivity to the zeptomole range, to design a cost-effective read out system, and to clinically validate this technology. This is important next step for the translation of barcoding technologies to the clinic.

Ming et al. Chan 
We expect the barcoded device to enable clinicians to better manage the spread of

70 diseases by improving the diagnosis of infectious pathogens, which can spread rapidly leading to economic burden, morbidity, and mortality. The integration of mobile-cellular devices with state-of-the-art multiplexing molecular diagnostic devices would help alleviate these problems by permitting clinical diagnosis in the absence of large laboratory infrastructure leading to more appropriate treatment, as well as enable real-time global surveillance of infectious transmission

75 events and predict temporal infection trends through crowd-sourced data collection. While the concepts of smartphones have been proposed for diagnostic applications, they have primarily been used in direct imaging applications from identifying bacteria or viruses labeled with a fluorophore, ${ }^{17,18}$ cell counting, ${ }^{19-21}$ to imaging the test lines on lateral flow immunoassays ${ }^{22}$ and results from custom-made lab-on-chips assays, ${ }^{23-25}$ among many other approaches ${ }^{26,27}$ Many of

80 these techniques often cannot diagnose the early stages of infection because of poor analytical sensitivity. More importantly they are incapable of detecting different strains or pathogens in a high-throughput manner due to their inability to detect multiple biomarkers simultaneously. To validate our technology, we demonstrate the ability to detect patients infected with the multiple chronic blood-borne infections such as HIV and hepatitis B because these co-infections can

85 accelerate disease progression. The diagnostic device can be extended to develop molecular testing panels for other important pathogens underlying sexually transmitted infections, malaria, influenzas $\mathrm{A}$ and $\mathrm{B}$, and tuberculosis by simply modifying the barcode recognition molecules to the target of choice.

\title{
RESULTS AND DISCUSSION
}

\section{Integration of quantum dot barcoding with smartphone technology}

\author{
Ming et al. Chan
}


A multiplex-chip platform that is simple to use and can be easily transported were two of the main design foci of our diagnostic device. To this end, we arrayed the quantum dot barcodes on microfabricated slides with controlled number of microbeads per unit area for detecting genetic samples (Figure 1a). Barcodes in a chip format are easier to transport than when they are

95 in solution. And compared to microarrays where the assay is performed on a flat surface, a barcoded chip is cheaper to produce due to cheaper microbead production, offers faster reaction kinetics due to the increased surface area, and allows higher tunability of microbead size and conjugation chemistry. ${ }^{28}$ Microbead arrays on a chip are currently used in sequencing analysis but the cost of the final chip is high because the microbeads are arrayed on the ends of optical

100 fibers. ${ }^{29}$ We developed a simple method to array quantum dot barcodes on the surface of a chip. Glass slides were microfabricated with $3.0 \mu \mathrm{m}$-diameter wells (Figure 1b). A solution of $\sim 3.0$ $\mu \mathrm{m}$ sized microbead barcodes containing different combinations of fluorescence emitting $\mathrm{CdSeS}$ alloyed-ZnS quantum dots were added to the chip. They spontaneously settled into each well, reducing overlapping and aggregation that may confound analysis of their fluorescence in later

105 steps (Figure 1c). Once bound, these microbeads do not desorb from the surface as they are held in place by non-covalent forces. The concentration and size of the barcodes determine the filling efficiency (Supporting Information Figure S1). While minor variations of microbead numbers from chip to chip were expected, they would constitute only a fraction of the thousands of microbeads functionalized on each chip, and thus not have a significant effect on detection 110 outcomes. The deposition of barcodes on the chip, compared to those stored in solution, enables higher portability of barcodes and reduces the number of steps in the quantum dot barcode assay process. The device itself is also portable and easy to use (Figure 1d). The components batteries, switch, laser diodes, lenses, and filters - and 3D-printed plastic chassis are all Ming et al. Chan 
lightweight and able to fit in one hand. The two laser diodes are switched on independently via a

115 manual switch: Excitation Laser $1(405 \mathrm{~nm})$ excites the barcodes and Excitation Laser 2 (650 $\mathrm{nm})$ excites the secondary label with a filter $\left(\lambda_{\mathrm{ex}}=655 / 15\right)$ that controls the wavelength excitation observed by the chip. The eyepiece and movable objective lens magnifies and focuses barcodes on the chip to allow them to be viewable clearly by the naked eye on the smartphone display. The smartphone camera, in this case Apple's iPhone 4S, then captures that view. A total 120 of five images are acquired for each sample, corresponding to each of the five emission filters $\left(\lambda_{\mathrm{em}}=430 \mathrm{LP}, 530 / 10,580 / 10,640 / 10\right.$, and 692/40). Specifically, the 430LP filter image is used for extracting the location and size of the barcodes in the camera field-of-view; the 530/10, $580 / 10$, and $640 / 10$ filter images for isolating quantum dot signals to determine barcode signatures; and the 692/40 filter image for isolating the secondary label signal to determine the 125 presence of target analyte bound to the barcode surface. While quantum dot barcode differentiation using just one color image (i.e. $\lambda_{\mathrm{em}}=430 \mathrm{LP}$ filter image) is possible when the barcode colors are few and distinct (e.g. 'green', 'yellow', 'red'), it becomes increasingly difficult and prone to errors when higher multiplexing is desired, which requires barcodes to contain varying mixtures of the same quantum dots. This causes some barcodes to share similar

130 color channel intensities (Supporting Information Figure S2), increasing the possibility of false identifications. Our approach of using multiple emission filters, on the other hand, allows differentiation with high accuracy while remaining easily scalable for greater barcode combinations. Finally, a custom-written algorithm analyzes these images and produces the results. The algorithm develops a histogram of the optical signal from the secondary probe of all

135 barcodes within the sample. A threshold is established in a measurement based on the highest signal from barcodes that do not contain any of the target molecules of interest (i.e. the negative Ming et al. Chan 
control in the experiments). A graph of barcode numbers above this threshold is used to determine a positive or negative detection (Supporting Information Figure S3). We expect samples that have the target molecule of interest to have more barcodes above this threshold. We

140 chose this method of analysis rather than using absolute intensity values because we found this method to obtain greater consistency in the analysis due to reduction in skewing of the measurements from a small population of microbeads. Our measurement strategy is adapted from flow cytometry, where the quantification is based on single cell counts instead of a population average. The entire imaging and analytical process takes less than ten minutes and

145 can be performed with minimal training.

\section{Use of quantum dots over traditional fluorophores}

The unique optical properties of the quantum dots for barcoding are critical for engineering a low-cost multiplex device. Here we compared the optical properties of two microbeads encapsulated with organic fluorophores ('Yellow' with $\lambda_{\mathrm{em}}=480 \mathrm{~nm}$, and 'Nile Blue' with $\lambda_{\mathrm{em}}=630 \mathrm{~nm}$ ) versus quantum dots ('QD540' with $\lambda_{\mathrm{em}}=530 \mathrm{~nm}$ and 'QD640' with $\left.\lambda_{\mathrm{em}}=640 \mathrm{~nm}\right)$. As shown in Figure 2, the quantum dots have a continuous absorption profile while the organic fluorophore has a peak-like profile (Figure 2 'Spectra'). The absorbance profile presents a significant advantage for quantum dots for engineering point-of-care device. This would reduce the size of the final device and reduce costs as a quantum dot-barcode device will

155 only require a single energy source to excite all of the barcodes (in this case, we used a $405 \mathrm{~nm}$ diode laser) while the organic fluorophore-barcodes will require multiple emitting diodes or lasers to maximally excite different barcodes (Figure 2 'Image'). The quantum dot barcodes also have narrower emission profiles (e.g. full-width half-max of $\sim 35 \mathrm{~nm}$ versus $60 \mathrm{~nm}$ for 'QD540'

Ming et al. Chan 
and 'Yellow', respectively) and retain their emission peaks despite being excited by different

160 wavelengths (e.g. 'QD640' versus 'Nile Blue') (Figure 2 'Varying Excitation'). The narrower emission profiles allow more quantum dots, compared to board-emission fluorophores, to be used within a range of wavelengths (e.g. the $400-700 \mathrm{~nm}$ visible spectrum) without spectral overlap, enabling greater number of barcodes to be engineered for multiplexing analysis. This and the emission peak retention property also reduce the likelihood of false barcode

165 identification. Finally, the quantum dot barcodes are much more resistant to photobleaching compared to the organic fluorophore encapsulated barcodes (Figure 2 'Photobleaching'). This is important for accurate barcode identification as bleaching of the coded microbeads can lead to mis-detection. Using quantum dots for barcoding simplifies the read-out device and reduces the costs of the final device so that it could be broadly used in the future in both resource-rich and 170 resource-limited settings.

\section{Synthetic targets to assess device sensitivity and multiplexing capability}

The current detection platforms for identifying quantum dot barcodes require expensive instruments and detectors. ${ }^{14,15}$ Here we evaluated whether our device can differentiate the optical signals between barcodes as well as the secondary fluorescent probe used in our genetic assays.

175 Figures $3 \mathrm{a}$ and $\mathrm{b}$ demonstrate that an iPhone camera is able to capture the distinct optical emissions of each barcode on the microwell chip and proper filtering can differentiate the barcode optical signal from the secondary probe's signal. These studies confirmed that an iPhone camera can image barcodes on the chip surface and be used as a detector for biological assays.

We first determine the analytical performance of the microbead-based sandwich assay 180 using our engineered smartphone reader. We designed seven barcodes for detecting seven Ming et al. Chan 
infectious disease biomarker targets as shown in Table 1 plus two barcodes for the positive and negative control samples. When the target is absent (i.e. negative detection), the optical signal from the microbead comprises only the quantum dot signal of the barcode. When the target is present (i.e. positive detection), the microbead optical signal consists of emissions from both the 185 quantum dots and Alexa Fluor 647 dye secondary probe. The limit of detection and linear dynamic range for each of the targets for HIV, hepatitis B virus (HBV) and hepatitis C virus (HCV), and the influenza type A targets H1N1, H3N2, H5N1, and influenza type B (Flu B) (Figure $3 \mathrm{c}$ and $3 \mathrm{~d})$ is between 10 to $50 \mathrm{fmol}\left(6 \times 10^{9}\right.$ to $3 \times 10^{10}$ copies) and up to 40 -fold, respectively, in a final hybridization sample volume of $10 \mu \mathrm{L}$. This suggests that analytical

190 performance is independent of the infectious disease targets. Note that while the dye does photobleach under continuous excitation in the device, it still retains $>85 \%$ of its intensity within the first minute of excitation (Supporting Information Figure S4), the longest possible image acquisition time during our experiments, and thus should not have a significant impact on the sensitivity of detection.

Next, we demonstrated the ability to simultaneously detect multiple synthetic genetic targets from bloodborne virus panel (Figure 4). We prepared six different mock genetic samples by mixing various combinations of the genetic target sequences for each of the three pathogens of interest - HIV, HBV, and HCV - plus a positive control sequence to ensure that the barcodes are working as designed, and the secondary fluorescent probe sequence. For example, we would 200 prepare solutions that were spiked with the target sequences for HIV and positive control sequence in one combination (Figure 4b), then HIV, HBV, HCV and positive control sequence in another combination (Figure 4f). A final hybridization sample of $20 \mu \mathrm{L}$ was added to the chip and incubated at $37^{\circ} \mathrm{C}$ for 20 minutes, rinsed with a washing buffer, dried, imaged, and analyzed Ming et al. Chan 
using the algorithm. In all cases the target sequences were correctly identified by the assay. For

205 example, in our solution containing the sequences for HCV and positive control (Figure 4d), the bar graph shows our technique can discriminate between barcodes bound with secondary probes (i.e. HCV and positive control) versus those not bound (i.e. HIV, HBV and negative control). All of the probe recognition sequences for the bloodborne viral panels were carefully designed in silico to minimize cross-reactivity with closely related viruses using subtypes against the gag

210 gene of $\mathrm{HIV}$, core protein gene of $\mathrm{HBV}$ and nucleocapsid protein gene of $\mathrm{HCV}$, as per gold standard testing standards.

\section{Clinical validation of diagnostic device}

We determined whether the device was capable of detecting and differentiating samples from patients with HIV and HBV from those without (i.e., HIV- or HBV-negative subjects). The

215 experiment done using synthetic targets showed that the device has a limit of detection of $10^{-15}$ mol but to use the device for diagnosing patient samples, the limit of detection needed to be lowered by a factor of $10^{6}$. We cannot incorporate conventional PCR amplification methods to our device between these PCRs are bulky and expensive. To address this issue, we optimized an isothermal amplification step, which can be performed in $10-30$ minutes, is simple, and does not require precise temperature control.

Patient samples were collected using standard protocols and we extracted the hepatitis B viral DNA and HIV viral RNA using magnetic microbeads. The cell membrane was first disrupted by the addition of lysis buffer, and the surface functionalized magnetic microbeads captured viral nucleic acid. The sample was then placed in a magnetic separator to collect

225 magnetic microbeads. Before use in our assay, the HIV RNA was reverse-transcribed into cDNA. All samples were then amplified using recombinase polymerase amplification. The Ming et al. Chan 
recombinase proteins were added to the isolated genetic targets to form nucleoprotein complex, which facilitates strand-transfer at the homologous sequence of the template DNA. Singlestranded binding proteins then stabilize the displaced strand of the template DNA, and the DNA 230 polymerase extends the complimentary strand. ${ }^{30}$ Of the final amplified sample, $20 \mu \mathrm{L}$ of the double-stranded DNAs were denatured and added to the chip, incubated at $37^{\circ} \mathrm{C}$, rinsed with a washing buffer, dried, imaged, and analyzed using the algorithm in a manner that is similar to detection of the synthetic targets. Figure $5 \mathrm{a}$ and $5 \mathrm{~b}$ demonstrate the successful diagnosis of individual patient samples with HIV and HBV, respectively, of varying viral loads before

235 amplification (Supporting Information Table S1). These viral loads are reported as per a clinical setting, and represent a viral load range from treatment-naïve patients. They do not necessarily indicate the clinical limit of detection. The HBV-infected samples were comprised of multiple genotypes to ensure our test would be widely applicable. In order to determine whether the measurement results were significant, we combined the measurements from all samples of non-

240 infected subjects, and then compared them to the combined measurements from the infected patients with either HIV or HBV (Figure $5 \mathrm{c}$ and $5 \mathrm{~d}$, respectively). Our results showed a significant difference with a p-value of 0.05 and 0.01 , respectively. We further confirmed the results using flow cytometry (Supporting Information Figure S5) and showed comparable outcomes. Our diagnostic device is capable of detecting viral loads in the range of $10^{3}$ to $10^{9}$ 245 copies $/ \mathrm{mL}$ and different genotypes (Supporting Information Table S1). To confirm our device is capable of multiplex detection of patient samples, we mixed $10 \mu \mathrm{L}$ of each amplified patient samples containing HIV and/or HBV. We performed four-plex assays (HIV, HBV, as well as positive and negative controls), and the results of which clearly demonstrated that the device is capable of differentiating between the two viruses (Figure 5e-h using smartphone device, Ming et al. Chan 
250 Supporting Information Figure S6 using flow cytometry). We further investigated the level of specificity with our genetic assay by sequencing $7 \mathrm{HBV}$ and $10 \mathrm{HIV}$ patient samples (Supporting Information Figure S7 and S8, respectively). We found that there are 5 to 9 and 3 to 4 mismatches within HBV and HIV probe-binding regions used for the assay, respectively. Although we acknowledge that we have only used wild-type probes to detect patient samples in

255 this study, we believe that the level of specificity can be further improved to detect single base pair mismatch by following Liong, M. et al's approach of using mutant probes that are designed specifically to match mutated region and therefore bind stronger with mutated target than the wild-type target. ${ }^{31}$ The development of our device for diagnosing single-nucleotide polymorphisms was not a focus but such studies will be a focus in future studies. Our clinical

260 validation with real-world HBV- and HIV-infected patient samples demonstrates the effectiveness of the proposed detection platform for diagnosing infectious diseases.

All of the pathogen targets used as test panels in this study represent major infectious threats to the global community, necessitating the development of effective and innovative means for detection and informatics to identify infected individuals and accelerate clinical

265 management. HIV, HBV and HCV are prevalent in resource-limited settings and pose major threats to populations, often related to unknown transmission through sexual contact, drug use and contaminated blood products. ${ }^{32,33}$ For more rapidly spreading pathogens, immediate implementation of infection control measures and enhanced surveillance to curb the spread of disease will be critical. Here we have shown that the integration of quantum dot barcodes with 270 smartphone technology can be used for multiplex molecular diagnosis of these infectious diseases with wireless transmission. The steps in our assay, excluding the standard blood collection/separation processes, there are four-steps (1 through 4) and two-steps (only 3 and 4)

Ming et al. Chan 
the genetic and proteomic detection process respectively. They are (1) extraction of the genetic target, (2) amplification of the target, (3) recognition and hybridization to barcodes on chip and

275 secondary probe, and (4) read-out of the chip optical signal with the smartphone followed by analysis. We believe these two or four steps could be automated in black box system. We envision a final blackbox device to all of these components, with specific chambers for each of these steps and their overall dimensions (Figure 6). Each compartment would contain disposable lyophilized samples that can be dissolved by buffers, and transferred from one compartment to 280 the next using capillaries and electrically-driven flow. Building a single unit device is conventionally done as technologies advance from academic development to commercial use once each step in the process has been confirmed to work as design. The device may also be custom-designed for different types of target molecules. For example, in detection of a protein target, we would not need steps 1 and 2 . The number of device chambers in the device can be

285 engineered according to the target molecule. This diagnostic device should be both easy-to-use and transportable and enable wireless transmission of diagnostics for interpretation, thus allowing the mapping, surveillance, and potentially prediction of diseases in real-time. The chip is highly versatile for proteomic, genomic, or whole pathogen targets. ${ }^{7,16}$ It may also be designed to detect different pathogen strains, co-infections, markers of drug resistance, or diagnose 290 infections that are difficult to differentiate clinically due shared symptoms such as a fever or rash. The only requirement will be to design panels of barcodes for each specific infectious disease agent. Future work will focus on integrating the extraction and amplification components with the existing device into a singular unit, field-testing of the device, and to conduct a clinical trial with greater sample size to evaluate clinical sensitivity and specificity in both the developed 295 and developing worlds. Although we have conducted our studies using a specific brand of Ming et al. Chan 
smartphone, the concept lends itself to further device engineering such that it can physically accommodate any cellphone or smartphone. This device can be assembled into a point-of-care unit that will enable hospitals, disease control centers, mobile units and the military to monitor and potentially predict the onset, spread, and resistance of infectious diseases.

METHODS

\section{Quantum Dot Synthesis}

Quantum dots (CdSeS alloyed-ZnS capped) of peak emission wavelength $540 \mathrm{~nm}$ (“QD540”) were purchased from CytoDiagnostics and used as instructed. Quantum dots of peak emission wavelengths $515 \mathrm{~nm}$ (“QD515”), $547 \mathrm{~nm}$ (“QD547”), $560 \mathrm{~nm}$ (“QD560”), $589 \mathrm{~nm}$

305 (“QD589”), $596 \mathrm{~nm}$ (“QD596”), $615 \mathrm{~nm}$ (“QD615”), and $640 \mathrm{~nm}$ (“QD640”) were synthesized and characterized according to published procedures $\mathrm{s}^{34,35}$ and stored in chloroform at room temperature until later use.

\section{Quantum Dot Barcode Synthesis}

Quantum dot barcodes were prepared by mixing together the quantum dots (QD515, 310 QD540, QD547, QD560, QD589, QD596, QD615 and QD640) in different ratios with a polymer-based solution (Supporting Information Table S2 and S3). The polymer solution consisted of poly(styrene-co-maleic anhydride) (32\%, cumene terminated) from Sigma-Aldrich dissolved in chloroform, with the polymer concentration at 4-wt $\%$. The resultant quantum dot polymer solution was then introduced into a nozzle system from Ingeniatrics using a syringe

315 pump from Harvard Apparatus at a rate of $0.9 \mathrm{~mL} / \mathrm{hour}$, as well as double-distilled (DD) water as the focusing fluid at a rate of $180 \mathrm{~mL} /$ hour. The nozzle system was then submerged inside a

Ming et al. Chan 
beaker partially filled with double distilled water. The polymeric barcode microbeads were synthesized in situ, and the microbeads formed a white colloidal suspension in the water. After synthesis, the valve was closed and the microbeads were stabilized by overnight stirring and then

320 collected. The microbeads were filtered using $35 \mu \mathrm{m}$ BD Falcon nylon mesh strainer cap, and characterized using an automated Beckman Coulter Vi-Cell counter, and stored in DD water at 4 ${ }^{\circ} \mathrm{C}$ until use. The quantum dot concentrations required for preparing the barcodes are presented in Supporting Information Table S2 and S3.

\section{Excitation, Absorption, and Emission Spectra Measurement}

The excitation and emission spectra of the Yellow and Nile Blue microbeads were measured using the Excitation and Emission Acquisition modes, respectively, on Horiba Jobin Yvon FluoroMax-3 fluorometer. The quantum dot absorption spectra were measured using Shimadzu UV-1601PC UV-Visible spectrophotometer. The quantum dot barcode emission spectra were measured using the Emission Acquisition mode on Horiba Jobin Yvon FluoroMax-

3303 fluorometer.

\section{Sensitivity Assay}

Sensitivity assays (Figure 3c and 3d) were performed directly on the microwell chips for all infectious disease DNA target strands $\left(\mathrm{T}_{-} \mathrm{H} 1 \mathrm{~N} 1, \mathrm{~T}_{-} \mathrm{H} 3 \mathrm{~N} 2, \mathrm{~T} \_\mathrm{H} 5 \mathrm{~N} 1, \mathrm{~T}_{-}\right.$FluB, T_HIV, T_HBV, and T_HCV) and their respective conjugated barcode microbeads (B_H1N1, B_H3N2, 335 B_H5N1, B_FluB, B_HIV, B_HBV, and B_HCV). DNA target strands from Bio Basic Inc., purchased HPLC-purified and used without further purification, were prepared in increasing concentrations of $0,5,10,50,100,500,1000$, and $2000 \mathrm{fmol} / \mu \mathrm{L}$ in TE buffer. DNA detection strand from IDT DNA Technologies with Alexa647 fluorophore on the 5' end, purchased HPLCMing et al. Chan 
purified and used without further purification, were prepared with concentration of $100 \mathrm{pmol} / \mu \mathrm{L}$

340 in TE buffer. Both DNA target and detection strand samples were stored at $4{ }^{\circ} \mathrm{C}$ until further use. To perform the assay, $1 \mu \mathrm{L}$ of the conjugated microbead sample, corresponding to approximately $10^{4}$ conjugated microbeads, was deposited on a microwell chip for each assay condition and let dry for 1 hour. Then, $1 \mu \mathrm{L}$ of each DNA target strand sample was mixed with $5 \mu \mathrm{L}$ of hybridization buffer (10x SSC, $0.1 \%$ SDS, heated to $60{ }^{\circ} \mathrm{C}$ ), $3 \mu \mathrm{L}$ of DD water, and $1 \mu \mathrm{L}$ of

345 DNA detection strands or DD water (for the blank condition). This resulted in a total hybridization volume of $10 \mu \mathrm{L}$ for each assay condition, which include blank, $0,10,50,100$, 300, 500, 1000, and 2000 fmol target DNA. The hybridization solution for each assay condition was deposited over the conjugated microbead spots on the microwell chips and incubated at 37 ${ }^{\circ} \mathrm{C}$ for 20 minutes. The microwell chips were then submerged in $10 \mathrm{~mL}$ of washing buffer $(0.5 \mathrm{x}$

$350 \mathrm{SSC}, 0.1 \% \mathrm{SDS}$, heated to $37^{\circ} \mathrm{C}$ ), washed by agitation for $20 \mathrm{~s}$, then let dry for 5 minutes before being imaged. Note that care must be taken so that the washing buffer does not dry and crystallize over the sample spots.

\section{Multiplexing Assay}

Cross reactivity between the bloodborne virus DNA target strands (T_HIV, T_HBV, and

355 T_HCV) and their corresponding conjugated barcodes (B_HIV, B_HBV, and B_HCV), as well as positive and negative control cases (B_Pos and T_Pos, and B_Neg and T_Neg, respectively), was studied (Figure 4). First, $6 \mu \mathrm{L}$ of each conjugated barcode sample, corresponding to approximately $6 \times 10^{4}$ barcodes each, were mixed together with $90 \mu \mathrm{L}$ of DD water to produce a $4 \mathrm{x}$ dilution factor of the original. The dilution was to reduce microbead aggregation after 360 deposition on chip, which may confound barcode resolution during analysis. To perform the Ming et al. Chan 
assay, $8 \mu \mathrm{L}$ of the diluted conjugated barcode mixture, corresponding to approximately $2 \times 10^{4}$ conjugated microbeads, was deposited on a microwell chip for each multiplexing case and let dry for 4 hours. Then, $4 \mu \mathrm{L}$ of each target case (DD water for the negative conditions, and corresponding DNA target strand sample with concentration of $2 \mathrm{pmol} / \mu \mathrm{L}$ for the positive conditions) was mixed with $40 \mu \mathrm{L}$ of hybridization buffer (10x SSC, $0.1 \%$ SDS, heated to $60{ }^{\circ} \mathrm{C}$ ) and $20 \mu \mathrm{L}$ of the detection strand (concentration of $100 \mathrm{pmol} / \mu \mathrm{L}$ ). This resulted in a total hybridization volume of $80 \mu \mathrm{L}$ for each multiplexing case. From this, $20 \mu \mathrm{L}$ of the hybridization solution for each multiplexing case was deposited over the conjugated barcode spots on the microwell chip and incubated at $37^{\circ} \mathrm{C}$ for 20 minutes. The microwell chip was then submerged

370 in $10 \mathrm{~mL}$ of washing buffer $\left(0.5 \mathrm{x}\right.$ SSC, $0.1 \%$ SDS, heated to $\left.37^{\circ} \mathrm{C}\right)$, washed by agitation for 20 $\mathrm{s}$, washed again in another $10 \mathrm{~mL}$ of washing buffer to further reduce non-specific binding, and then let dry for 5 minutes before being imaged. Note that care must be taken so that the washing buffer does not dry and crystallize over the sample spots.

\section{Whole Blood Collection/Separation, Viral DNA/RNA Extraction, and Reverse} Transcription

Whole blood was collected by venipuncture in either a Vacutainer (serum) or anticoagulant-treated tubes (plasma). Tubes were inverted several times, and stood upright for 30 - 60 minutes (for serum collection). Samples were then spun in a refrigerated centrifuge, and serum or plasma was aliquoted and stored at $-80{ }^{\circ} \mathrm{C} . \mathrm{HBV}$ or HIV nucleic acid was extracted using the Chemagic Viral DNA/RNA Kit (PerkinElmer), and HIV RNA was then reverse transcribed as per the iScript cDNA Synthesis kit (Bio-Rad).

\section{Recombinase Polymerase Amplification (RPA) and Purification}

Ming et al. Chan 
RPA was performed using either extracted DNA or reverse-transcribed cDNA using the TwistAmp Basic kit (TwistDx, UK). For HBV detection, a premix solution containing 0.48

$\mathrm{pmol} / \mu \mathrm{L}$ of each forward and reverse primers (5'GGCATGGACATTGACCCTTATAAAGAATTTGG-3', $\quad$ 5'TGTCGAGAAGGTCCCGAATAGACGGAAAGA-3'), $9.2 \mu \mathrm{L}$ of nuclease-free water, $29.5 \mu \mathrm{L}$ of rehydration buffer, and $4 \mu \mathrm{L}$ of the either extracted non-infected or infected DNA was prepared in a volume of $47.5 \mu \mathrm{L}$. This solution was then transferred to a tube containing the 390 reaction pellet and mixed. The reaction was initiated by the addition of $2.5 \mu \mathrm{L}$ of $280 \mathrm{mM}$ magnesium acetate, and incubated at $37^{\circ} \mathrm{C}$ for 10 minutes to produce 100 base-pair amplicon.

For HIV detection, a premix solution containing $0.48 \mathrm{pmol} / \mu \mathrm{L}$ of each forward and reverse primers (5'-GAAAGGTGAAGGGGCAGTAGTAATACAAGACA-3', 5' CCACACAATCATCACCT GCCATCTGTTTTCCA-3'), $11.2 \mu \mathrm{L}$ of nuclease-free water, 29.5

$395 \mu \mathrm{L}$ of rehydration buffer, and $2 \mu \mathrm{L}$ of the either extracted and reverse-transcribed non-infected or infected cDNA was prepared for a total volume of $47.5 \mu \mathrm{L}$. This solution was then transferred to a tube containing the reaction pellet and mixed. The reaction was initiated by the addition of 2.5 $\mu \mathrm{L}$ of $280 \mathrm{mM}$ magnesium acetate, and incubated at $37^{\circ} \mathrm{C}$ for 30 minutes to produce 116 basepair amplicon.

RPA products were purified using EZ-10 Spin Column DNA Gel Extraction Kit (Bio Basic), and eluted into $50 \mu \mathrm{L}$ for detection. Purified DNA was visualized by gel electrophoresis, and kept at $4{ }^{\circ} \mathrm{C}$ until later use.

\section{Mono-Infection Assays using Amplified Clinical Samples}

Ming et al. Chan 
Clinical mono-infection assays (Figure 5a-d) were performed directly on the microwell

405 chips using HIV- and HBV-negative, as well as HIV- and HBV-positive samples after amplification. DNA detection strands from IDT DNA Technologies with Alexa647 fluorophore on either 5' end (CD_HIV) or 3' end (CD_HBV), purchased HPLC-purified and used without further purification, for the HIV and HBV target sequences, were prepared with concentration of $100 \mathrm{pmol} / \mu \mathrm{L}$ in TE buffer and stored at $4{ }^{\circ} \mathrm{C}$ until further use. To perform the assay, $1 \mu \mathrm{L}$ of the

410 conjugated microbead sample, corresponding to approximately $10^{4}$ conjugated microbeads, was deposited on a microwell chip for each assay condition and let dry for 1 hour. During this time $20 \mu \mathrm{L}$ of the amplified sample was mixed with $5 \mu \mathrm{L}$ of the corresponding detection strand and denatured at $100{ }^{\circ} \mathrm{C}$ for 15 minutes. Then, the $25-\mu \mathrm{L}$ denaturation solution was mixed with 25 $\mu \mathrm{L}$ of hybridization buffer (10x SSC, $0.1 \%$ SDS, heated to $60{ }^{\circ} \mathrm{C}$ ). The $50-\mu \mathrm{L}$ hybridization

415 solution was deposited over the dried conjugated microbead spot on the microwell chip and incubated at $37^{\circ} \mathrm{C}$ for 60 minutes, and let cool at room temperature for 5 minutes. The microwell chip was then submerged in $200 \mathrm{~mL}$ of washing buffer ( $0.5 \mathrm{x}$ SSC, $0.1 \%$ SDS, heated to $37^{\circ} \mathrm{C}$ ), washed by agitation for $10 \mathrm{~s}$, washed again in another $200 \mathrm{~mL}$ of washing buffer to further reduce non-specific binding, and let dry for 5 minutes before being imaged. Note that care must 420 be taken so that the washing buffer does not dry and crystallize over the sample spots.

\section{Co-Infection Assays using Amplified Clinical Samples}

Cross-reactivity between the amplified HIV and HBV clinical samples (CT_HIV and CT_HBV) and their corresponding conjugated barcodes (CB_HIV and CB_HBV), as well as positive and negative control cases (CB_Pos and CT_Pos, and CB_Neg and CT_Neg,

425 respectively), was studied (Fig $5 \mathrm{e}-\mathrm{h}$ ). First, $5 \mu \mathrm{L}$ of each conjugated barcode sample, Ming et al. Chan 
corresponding to approximately $5 \times 10^{4}$ barcodes each, were mixed together with $20 \mu \mathrm{L}$ of DD water to produce a $2 \mathrm{x}$ dilution factor of the original. The dilution was to reduce microbead aggregation after deposition on chip, which may confound barcode resolution during analysis. To perform the assay, $1 \mu \mathrm{L}$ of the diluted conjugated barcode mixture, corresponding to

430 approximately $5 \times 10^{3}$ conjugated microbeads, was deposited on a microwell chip for each multiplexing case and let dry for 1 hour. During this time $10 \mu \mathrm{L}$ of each amplified sample was mixed with $5 \mu \mathrm{L}$ of each of the corresponding detection strands $(5 \mu \mathrm{L}$ of CD_HIV, $5 \mu \mathrm{L}$ CD_HBV , and $10 \mu \mathrm{L}$ of $\mathrm{CD}$ for both CT_Pos and CT_Neg) and denatured at $100{ }^{\circ} \mathrm{C}$ for 15 minutes. Then, the $60-\mu \mathrm{L}$ denaturation solution was mixed with $60 \mu \mathrm{L}$ of hybridization buffer

435 (10x SSC, $0.1 \%$ SDS, heated to $60{ }^{\circ} \mathrm{C}$ ). The $120-\mu \mathrm{L}$ hybridization solution was deposited over the dried conjugated microbead spot on the microwell chip and incubated at $37{ }^{\circ} \mathrm{C}$ for 60 minutes, and let cool at room temperature for 5 minutes. The microwell chip was then submerged in $400 \mathrm{~mL}$ of washing buffer $\left(0.5 \mathrm{xSC}, 0.1 \% \mathrm{SDS}\right.$, heated to $\left.37^{\circ} \mathrm{C}\right)$, washed by agitation for $10 \mathrm{~s}$, washed again in another $400 \mathrm{~mL}$ of washing buffer to further reduce non-

440 specific binding, and let dry for 5 minutes before being imaged. Note that care must be taken so that the washing buffer does not dry and crystallize over the sample spots.

\section{Device Design and Construction}

The device was designed using SolidWorks 2012 and 3D printed commercially (Reprodux, North York, Ontario, Canada). Laser diode excitation sources of $405 \mathrm{~nm} 50 \mathrm{~mW}$ 445 (http://www.ebay.com/itm/170719374707), and $650 \mathrm{~nm} 50 \mathrm{~mW}$ (http://www.ebay.com/itm/1pcs650nm-50mw-Red-Laser-Diode-Dot-Module-

1370650098149?pt=LH_DefaultDomain_0\&hash=item564c77a9e5) were purchased online and Ming et al. Chan 
secured into the device as delivered. The device was designed such that both lasers could excite the same spot on the chip. An excitation filter $\lambda_{\text {ex }}=655 / 15 \mathrm{~nm}$ (Edmund Optics) was fixed in

450 front of the $650 \mathrm{~nm}$ laser diode source to reduce background signal. Both laser diodes were electrically connected to 2x AA batteries via a battery holder and single-pole triple-throw switch (both purchased from a local electronics shop) that switches between the two sources as well as an OFF state. A generic $160 x-200 x$ pocket microscope was purchased online (http://www.gadgetplus.ca/science/Microscope160-200x.html). It was disassembled to extract

455 the eyepiece and objective lenses, and installed into the device manually. The eyepiece was fixed in place but the objective was made to be movable long a track to allow focusing on the sample.

\section{Sample Imaging}

All images were acquired using the iPhone $4 \mathrm{~S}$ from Apple (unless otherwise specified) mounted in our device. Quantum dot barcodes and Alexa647 fluorophore were excited using

460 laser diodes of wavelengths $405 \mathrm{~nm}$ and $650 \mathrm{~nm}$, respectively. Emission filters $\lambda_{\mathrm{em}}=430 \mathrm{LP}$ (Thorlabs), $\lambda_{\mathrm{em}}=530 / 10$ (Thorlabs), $\lambda_{\mathrm{em}}=580 / 10$ (Thorlabs), $\lambda_{\mathrm{em}}=640 / 10$ (Thorlabs), and $\lambda_{\mathrm{em}}=$ 692/40 (Semrock, Brightline Cy5-4040A) were placed in the device's emission filter slot one at a time during imaging. The emission filter $\lambda_{\mathrm{em}}=430 \mathrm{LP}$ was used in conjunction with a neutral density filter $\mathrm{OD}=1.3$ (Thorlabs) to image all barcodes to determine their size and location, 465 while avoiding intensity saturation. The emission filters $\lambda_{\mathrm{em}}=530 / 10, \lambda_{\mathrm{em}}=580 / 10$, and $\lambda_{\mathrm{em}}=$ 640/10 corresponded with quantum dots QD540, QD589, and QD640, respectively, and were used to isolate for their fluorescence for resolving barcodes. The emission filter $\lambda_{\mathrm{em}}=692 / 40 \mathrm{was}$ used to isolate for the detection strand Alexa647 secondary label fluorescence as a means to measure the amount of analyte that hybridized with its corresponding capture strand. Image

Ming et al. Chan 
470 exposure times, made adjustable with the use of the NightCap app from Apple's App Store, was maintained at $1 \mathrm{~s}$ for all filters. In the case of fluorophore particles, they were excited using only the $405 \mathrm{~nm}$ laser diode source and imaged using only the emission filter $\lambda_{\mathrm{em}}=430 \mathrm{LP}$, the images of which were used for subsequence intensity analysis.

\section{Image Analysis}

475

A custom-made algorithm was written in MathWork's MATLAB for all image analysis (see supplement section for the code). The algorithm accepts as inputs five emission filter images $\left(\lambda_{\mathrm{em}}=430 \mathrm{LP}, \lambda_{\mathrm{em}}=530 / 10, \lambda_{\mathrm{em}}=580 / 10, \lambda_{\mathrm{em}}=640 / 10\right.$, and $\left.\lambda_{\mathrm{em}}=692 / 40\right)$ of a sample. The images were cropped to include microbeads of interest based on user selection. The cropped filter images were aligned with the $\lambda_{\mathrm{em}}=430 \mathrm{LP}$ filter image through the use of the discrete 480 Fourier transform registration. ${ }^{36,37}$ The algorithm then identified the size and location of each microbead, based on its appearances in the $\lambda_{\mathrm{em}}=430 \mathrm{LP}$ filter image, using the Hough transform. ${ }^{38,39}$ Erroneously identified microbeads (e.g. debris, imaging artefacts, overlapping microbeads) were excluded based on user input. Each microbead was then associated with the mean pixel intensity across its area at each of the four remaining filter images. For each microbead, the $\lambda_{\mathrm{em}}=530 / 10, \lambda_{\mathrm{em}}=580 / 10$, and $\lambda_{\mathrm{em}}=640 / 10$ filter image intensities comprised its intensity profile, while the $\lambda_{\mathrm{em}}=692 / 40$ filter image intensity indicated the fluorescent secondary probe intensity. In order to identify the microbeads on the chip, known barcode intensity profiles were first established (Supporting Information Figure S9). These profiles were obtained by imaging all the barcodes - B_H1N1, B_H3N2, B_H5N1, B_FluB, B_HIV, B_HBV, B_HCV, B_Pos, B_Neg, CB_HIV, CB_HBV, CB_Pos, and CB_Neg - alone (Table 1) and calculating the mean filter intensity across all microbeads for each filter. A microbead's intensity profile was

Ming et al. Chan 
then compared against each known barcode's intensity profile to identify the barcode of interest. Specifically, a barcode was classified according to its type (i.e. synthetic or clinical sample) and highest to lowest intensities among the filters $\lambda_{\mathrm{em}}=530 / 10, \lambda_{\mathrm{em}}=580 / 10$, and $\lambda_{\mathrm{em}}=640 / 10$. This 495 narrowed the selection down to either one barcode, in which case the barcode of interest was identified, or two barcodes. Between the two possibilities $B_{\text {high }}$ (with higher mean intensities) and $\mathrm{B}_{\text {low }}$ (with lower mean intensities) a threshold was defined for $\mathrm{B}_{\text {low }}$ :

$$
I_{\text {barcode }}=I_{\text {mean }}+I_{S T D}
$$

$I_{\text {barcode }}=$ Intensity threshold for $\mathrm{B}_{\mathrm{low}}$.

500

$$
\begin{aligned}
& I_{\text {mean }}=\text { Mean intensity of } \mathrm{B}_{\text {low }} \text {. } \\
& I_{S T D}=\text { Intensity standard deviation of } \mathrm{B}_{\text {low }} .
\end{aligned}
$$

This threshold was calculated for the highest intensity amongst the three filters $\lambda_{\mathrm{em}}=530 / 10, \lambda_{\mathrm{em}}$ $=580 / 10$, and $\lambda_{\text {em }}=640 / 10$ for $B_{\text {low }}$. If the highest intensities were similar in value between $B_{\text {low }}$ and $\mathrm{B}_{\text {high }}$, the second highest filter intensity was used. With this, if the microbead's corresponding filter intensity was equal to or lower than $I_{\text {barcode }}$, the barcode of interest was $\mathrm{B}_{\text {low }}$, otherwise $B_{\text {high }}$ was chosen. An example to illustrate the microbead identification process (Supporting Information Figure S10):

A microbead B is imaged during a synthetic sample multiplexing test - thus classified as 'synthetic sample' - has an intensity profile of $\left[\lambda_{\mathrm{em}}=530 / 10, \lambda_{\mathrm{em}}=580 / 10, \lambda_{\mathrm{em}}=640 / 10\right]=[4$, $51040,80]$, so $\left[\lambda_{\mathrm{em}}=640 / 10\right]>\left[\lambda_{\mathrm{em}}=580 / 10\right]>\left[\lambda_{\mathrm{em}}=530 / 10\right]$. On examination the set of barcodes used for synthetic multiplexing (Supporting Information Figure S9a), B has a similar intensity profile (i.e. same order of highest to lowest filter intensities) as B_Pos and B_Neg. Between Ming et al. Chan 
them, B_Neg has the lower mean intensities and thus chosen as $B_{\text {low }}$, with the its highest filter intensity at $\left[\lambda_{\mathrm{em}}=640 / 10\right]=30.50$. And because it is much lower and thus distinguishable from 515 B_Pos's $\left[\lambda_{\mathrm{em}}=640 / 10\right]=80.35$, the intensity threshold is calculated using B_Neg's $\left[\lambda_{\mathrm{em}}=\right.$ 640/10]:

$$
I_{\text {barcode }}=I_{\text {mean }}+I_{S T D}=30.50+6.26=36.76 \text {. }
$$

Since B's $\left[\lambda_{\mathrm{em}}=640 / 10\right]=80$ is greater than $I_{\text {barcode }}=36.76$ from B_Neg, B is identified as B_Pos.

We determined whether the analyte of interest is present by using the intensity values from the $\lambda_{\mathrm{em}}=692 / 40$ filter, which isolates for the Alexa Fluor 647 secondary probe signal. For the synthetic sample sensitivity assays the intensities were used directly to establish the limit of detection and dynamic range for the device. However, we used a micobead-counting method to determine the optical detection of multiplex samples and clinical samples because we achieve 525 greater accuracy in the measurement when the measurement is based on a comparison to negative controls. Negative and positive controls are always required in analyzing complex samples, as these control samples confirm whether a technique is working as designed. When we conduct the measurements, we develop a histogram of the fluorescence intensity from the secondary probe and compare that signal to those of the negative sample. Equation 2 describes this analysis.

$Q=$ (population of barcode whose $\lambda_{\mathrm{em}}=692 / 40$ filter intensity $\geq I_{\text {assay }}$ ) / (total barcode population) 
That is, the barcodes whose secondary probe intensities were equal to or above the threshold $I_{\text {assay }}$, defined empirically, were counted and a percentage, relative to the barcode's total

535 population, was calculated. In the case of multiplexed detection of synthetic bloodborne viral targets (Figure 4), a detection was considered positive if $Q>30 \%$ (i.e. over 30\% of said barcode had secondary probe signals above the threshold). In the case of amplified mono- and co-infected clinical samples (Figure 5), a detection was instead considered positive if $Q>3 \%$ due to their overall lower signals.

\section{Statistics and General Methods}

All data represent analysis from at least 100 barcodes to ensure they are representative of the experimental conditions studied. The variability in the number of barcodes analyzed is due to the field of view. In some cases, there are more microbeads per field of view than others. Once we analyze $>100$ barcodes, the measurements were relatively consistent and did not influence

545 the statistics. For the detection of mono-infected patient samples, we used infection-negative subject samples as a negative control, which is procedurally and clinically more accurate as negative controls than any other types of samples. To ensure clinical sample blindness, they were prepared and provided unlabeled by the collaborators (MJB, MO, and JJF) to the investigators (KM, JK, and WCWC), who then performed the experiments without knowledge of the samples'

550 identities. For where statistical tests were necessary (i.e. comparing the infection-negative subject groups with the HIV- or HBV-infected subject groups), the two-sided $t$-test was used because the data exhibits normal distribution (e.g. Supporting Information Figure S3).

\section{Human Subjects}

Ming et al. Chan 
The de-identified clinical samples were obtained from the Toronto Western Hospital

555 Liver Clinic and St. Michael's Hospital biobank repository. The protocol was approved by the Research Ethics Board of the University Health Network and St. Michael's Hospital, both affiliates of the University of Toronto. All patients provided written informed consent for storage and use of their specimens for research.

Ming et al. Chan 
Figure 1. Overview of the smartphone device utilizing quantum dot barcodes. (a) The assay involves the addition of patient samples to a chip coated with microbeads, which are optically barcoded by quantum dots and are coated with molecules that recognize a target analyte. This target analyte joins the barcode to the secondary probe. Since each barcode is conjugated with a

565 known bio-recognition molecule for a specific pathogen target, the imaging of the optical signal from the barcode would allow for the identification of the pathogen and whether it is present in a patient sample (i.e. lack of secondary probe signal indicates no pathogen present, in this case the yellow microbead). (b) A typical microwell chip containing different barcodes in each well. In a biological assay, we add a $20-\mu \mathrm{L}$ (for multiplexing synthetic targets), or a $50-\mu \mathrm{L}$ (for mono570 infection patient samples) total hybridization sample on the chip (see black arrow), incubate at $37^{\circ} \mathrm{C}$ for $20-60$ minutes, rinse, and image. (c) A smartphone camera captures the image of four different quantum dot barcodes arrayed on the surface of the chip. These barcodes are excited with a violet laser source $\left(\lambda_{\mathrm{ex}}=405 \mathrm{~nm}, 50 \mathrm{~mW}\right)$, optical signals collected by a set of lenses, filtered with $430 \mathrm{~nm}$ long-pass filter, and imaged using an Apple iPhone 4S smartphone with an

575 exposure time of $0.05 \mathrm{~s}$. (d) Two excitation sources excite the quantum dot barcoded chip independently. The optical emission is collected by a set of objective and eyepiece lenses, imaged using a smartphone camera, and interpreted as positive or negative detection using a custom-designed algorithm. The images may be sent wirelessly to a centralized facility for further evaluation or for the mapping and tracking of infectious diseases. (e) Image of the 580 smartphone device.

Figure 2. Comparison of the optical properties between organic dye molecules and quantum dot inside polystyrene microbeads. Polymeric particles impregnated with fluorophores ('Yellow' and 'Nile Blue') are compared with quantum dot barcodes ('QD540' and 'QD640'): excitation/absorption and emission spectra; visual images captured by our device when excited 585 using a $405 \mathrm{~nm}$ laser excitation source; emission spectra under varying excitation wavelengths; and photobleaching under continuous excitation in the device, representing the average intensities of 591, 642, 1198, and 1145 barcodes analyzed, over the 180 s duration, for 'Yellow', 'Nile Blue', 'QD540', and 'QD640', respectively.

Figure 3. Visual demonstration of a barcode assay and device assay sensitivity. (a) Yellow, 590 green, and red barcodes (identified as B_HBV, B_HCV, and B_Pos in Table 1, respectively) are deposited on the chip and imaged using the device $\left(\lambda_{\mathrm{ex}}=405 \mathrm{~nm}, \lambda_{\mathrm{em}}=430 \mathrm{LP}\right.$, exposure time $=$ $1 \mathrm{~s})$. (b) After the assay, the device-acquired fluorescence image of the microbeads bound with the target analyte and secondary probe $\left(\lambda_{\mathrm{ex}}=655 / 15, \lambda_{\mathrm{em}}=692 / 40\right.$, exposure time $\left.=1 \mathrm{~s}\right)$. Both green and red microbeads had positive signals. This demonstrates that their respective genomic 595 targets are present in the sample but not for the yellow barcode. (c) Sensitivity curves for genetic biomarkers for the bloodborne viruses human immunodeficiency virus (HIV), hepatitis B virus (HBV) and hepatitis C virus (HCV), representing 8017, 7324, 10334 barcodes analyzed, respectively. (d) Sensitivity curves for genetic biomarkers for the influenza A viruses H1N1, H3N5, and H5N1; and influenza B virus (FluB), representing 8491, 5967, 10182, and 4114 barcodes analyzed, respectively. All values represent the average secondary probe intensity, and

Ming et al. Chan 
error bars were calculated based on the standard deviation from three replications of each condition.

Figure 4. Multiplexed detection for synthetic target strands for the bloodborne viruses HIV, HBV, HCV with positive and negative controls. (a) Only positive control was present during 605 hybridization, representing 1039 barcodes analyzed. (b) Targets for HIV and positive control were present during hybridization, representing 1581 barcodes analyzed. (c) Targets for HBV and positive control were present during hybridization, representing 1749 barcodes analyzed. (d) Targets for HCV and positive control were present during hybridization, representing 1202 barcodes analyzed. (e) Targets for HIV, HBV, and positive control were present during 610 hybridization, representing 2401 barcodes analyzed. (f) All targets except for negative control were present during hybridization, representing 1343 barcodes analyzed. Results represent data from three experimental replications of each condition combined into a single data set. Note that samples above the dashed $30 \%$ line are considered positive detection, otherwise they are considered negative detection.

615 Figure 5. Detection of HIV and HBV in patient samples. (a) Detection of mono-infected amplified samples from $3 \mathrm{HIV}$-negative subjects and $10 \mathrm{HIV}$-positive patients, representing 1306, 728, 321, 1573, 626, 297, 1268, 429, 468, 499, 332, 375, and 287 barcodes analyzed, respectively, for each subject. (b) Detection of mono-infected amplified samples from $3 \mathrm{HBV}$ negative subjects and 7 HBV-positive patients, representing 361, 207, 1345, 371, 221, 1310, 806,

620665,778 , and 674 barcodes analyzed, respectively, for each subject. (c) Comparison between the average combined statistics of all subjects of the HIV-negative group (3 subjects) and HIVpositive group (10 patients) from (a); error bars represent standard deviation, with statistical significance $(P<0.05)$ indicated and determined using two-sided $t$-test. $(\mathbf{d})$ Comparison between the average combined statistics of all subjects of the HBV-negative group (3 subjects) and HBV-

625 positive group (7 patients) from (b); error bars represent standard deviation, with statistical significance $(P<0.01)$ indicated and determined using two-sided $t$-test. $(\mathbf{e}-\mathbf{h})$ Detection of coinfection assays simulated with amplified HIV- and HBV-negative, as well as HIV- and HBVpositive patient samples. (e) Only positive control was present during hybridization, representing 384 barcodes analyzed. (f) HIV-positive patient sample and positive control were present during

630 hybridization, representing 866 barcodes analyzed. (g) HBV-positive patient sample and positive control were present during hybridization, representing 1888 barcodes analyzed. (h) HIV- and HBV-positive patient samples, and positive control, were present during hybridization, representing 1019 barcodes analyzed. Results represent data from three replications of each condition combined into a single data set. Note that samples above the dashed 3\% line are

635 considered positive detection, otherwise they are considered negative detection. All samples were amplified. Additionally, all samples were blinded during the experiment to reduce bias.

Figure 6. Integrated assembled device. (a) A visual representation of the envisioned final device, with the different compartments colored for clarity, capillary tubes to show the flow of solutions between them, and a smartphone for scale. (b) Schematics of the envisioned final device with 640 colors matching the various compartments in (a) and numbers indicating the movement of sample in these compartments. (c) A flow diagram illustrating the steps required for detecting pathogen targets using the envisioned final device, from patient sample to imaging and analysis,

Ming et al. Chan 
with colors and numbers matching the compartments in (a) and (b). The liquid can be moved from one compartment to the next using electrically-driven flow.

Ming et al. Chan

$-30-$ 